\title{
Uterine torsion of 90 degrees: case report
}

\section{S. Tanouti*, M. Chakri, H. Taheri, H. Saadi, A. Mimouni}

Department of Obstetrics and Gynecology, Mohammed VI University Hospital Center, Oujda, Morroco

Received: 23 January 2020

Accepted: 28 February 2020

\section{*Correspondence:}

Dr. S. Tanouti,

E-mail: saloua.tanouti@gmail.com

Copyright: (C) the author(s), publisher and licensee Medip Academy. This is an open-access article distributed under the terms of the Creative Commons Attribution Non-Commercial License, which permits unrestricted non-commercial use, distribution, and reproduction in any medium, provided the original work is properly cited.

\begin{abstract}
Uterine torsion is defined as a rotation of uterus more than 45 degrees along its long axis. However, a pathologic rotation of the uterus beyond 45 degrees-torsion of the entire uterus-is rarely seen in obstetrical practice, authors report a case of torsion of the uterus by 90 degrees. The patient, a 30-year-old gravida 3 para 2 at 37 weeks' gestation with a singleton pregnancy, her prior obstetrical history included two uncomplicated term vaginal deliveries, and the current pregnancy had been uncomplicated until the date of presentation was admitted to the obstetrical unit with labour at 37 weeks 5 days ,on obstetric examination the patient was in labour with transversal presentation of the fetus so an emergency caesarean section (CS) was carried out for. At the time of CS, the diagnosis of uterine torsion of 90 degrees was made. After the delivery of the baby, uterus returned to anatomical position and the torsion corrected spontaneously. The patient recovered and was discharged home with her baby on the third postoperative day. Uterine torsion is an infrequently reported and potentially dangerous complication of pregnancy that occurs mainly in the third trimester.
\end{abstract}

Keywords: 90 degrees, Good recovery, Intraoperative diagnosis, Potentially dangerous, Rare, Uterine torsion

\section{INTRODUCTION}

Uterine torsion is defined as a rotation of uterus more than 45 degrees along its long axis. However, a pathologic rotation of the uterus beyond 45 degreestorsion of the entire uterus-is rarely seen in obstetrical practice, in this report a case of torsion of the uterus by 90 degrees. $^{1}$

\section{CASE REPORT}

This is a patient aged 30 years G3P2, the first two pregnancies are carried out with vaginal delivery without complications, pregnant with a pregnancy of 37 weeks.

The patient has no particular pathological history, her pregnancy had been uneventful throughout with no any other co morbidities admitted for uterine contractions. The examination on admission finds a patient who is hemodynamically and respiratory stable, obstetrical examination found a parturient in labour with a transversal presentation of the fetus. A preoperative assessment was initiated and the patient was rushed to the operating room for emergency caesarean section.

In the operating room, under rachi anesthesia, on intraop the diagnosis of torsion of uterus was made due to the round ligament and tubo ovarian complex of the left side were rotated to the right side and were lying anteriorly (Figure 1). Detorsion was attempted, uterus was detoded by 45 degrees; utmost care was taken to extend the uterine incision only to the right side so that uterine arteries lying anteriorly are not affected. Baby weighing $2.9 \mathrm{~kg}$ was born with Apgar scores were 10 at one minute and 10 at five minutes. After the delivery of the baby placenta was removed manually. Uterus was sutured in single layer and hemostasis ensured (Figure 2). The uterus had returned to its anatomical position following the delivery. Blood loss during the surgery was about 500 ml. No uterine anomalies or fibroids were seen. 


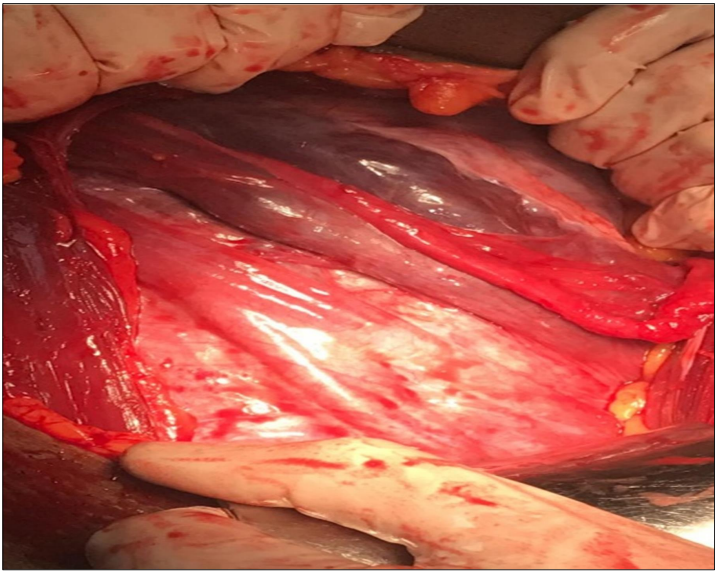

Figure 1: Torsion of uterus was made due to the round ligament and tube ovarian complex of the left side were rotated to the right side.

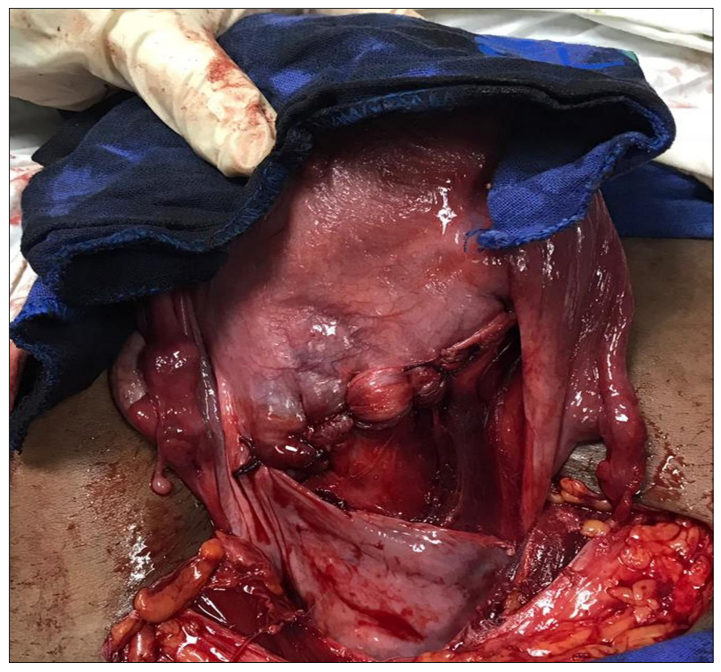

Figure 2: Sutured uterus.

The patient made an uneventful recovery and was discharged home on the third postoperative day with her baby.

\section{DISCUSSION}

The first report of uterine torsion in the human was published by Labbé in $1876 .{ }^{1,2}$ Since then, uterine torsion has been reported rarely, perhaps because of the nonspecific presentation and generally successful outcome for mothers. ${ }^{1,3,4}$ Nevertheless, it is a potentially dangerous complication of pregnancy.

The cause of uterine torsion in most cases is unknown. A case series by Piot et al, described a number of possible associative and causative factors: $32 \%$ cases had a fibroid uterus, $15 \%$ had other uterine anomalies such as a bicornuate uterus, $8 \%$ had pelvic adhesions, $7 \%$ had ovarian cysts or other adnexal masses, $5 \%$ had abnormal fetal presentation and fetal abnormalities were identified in $3 \%$ cases. In this series, over $30 \%$ cases had no cause identified. $^{5}$ Transverse fetal lie (or malpresentation) is commonly reported in association with uterine torsion, which is the case in the patient. ${ }^{6-9}$

More recent studies with no identified causative pelvic factors have suggested previous caesarean section as a common feature, and MRI of patients after caesarean section has suggested that poor isthmic healing, resulting in an elongated cervix with structural weakness and angulation may predispose to torsion of the uterus. Presentation of uterine torsion has been studied to be independent of maternal age, parity and gestation. Uterine torsion is difficult to diagnose preop since presenting symptoms and signs are non-specific and can mimic other situations like scar dehiscence and abruption placentae. Ultrasound can also help in diagnosing uterine torsion antenatal by the following features. ${ }^{10}$ Transposition of placental bed compared to previous scan and doppler recognition of ovarian vessels anterior to the uterus. MRI has been reported to help in diagnosing uterine torsion antenatally by the demonstration of $\mathrm{X}$ shaped configuration in the upper vagina. ${ }^{11}$ However in this case the patient was asymptomatic and hence this could not diagnose it antenatally.

Bilateral plication of round ligaments has been reported as a prophylactic measure to avoid recurrence of torsion.

\section{CONCLUSION}

Uterine torsion is an infrequently reported and potentially dangerous complication of pregnancy that occurs mainly in the third trimester.

Identification of anatomical landmarks can guide the surgeon to diagnose uterine torsion intra operatively and approach to the lower segment can be planned.

Detorsion must be attempted in all cases if possible.

Funding: No funding sources

Conflict of interest: None declared

Ethical approval: Not required

\section{REFERENCES}

1. Jovanovic D, Del Granado A, Stiller A. Torsion of the gravid uterus: a review and a case. J Reprod Med. 1972;8(2):81-4.

2. Biswas MK, Summers P, Schultis SA, Herrera EH, Pernoll ML. Torsion of the gravid uterus. A report of two cases. J Reprod Med. 1990;35(2):194-7.

3. Duplantier N, Begneaud W, Wood R, Dabezies C. Torsion of a gravid uterus associated with maternal trauma. A case report. J Reprod Med. 2002;47(8):683-5.

4. Jensen JG. Uterine torsion in pregnancy. Acta Obstet Gynecol Scand. 1992;71:260-5. 
5. Moores KL, Wood MG, Foon RP. A rare obstetric emergency: acute uterine torsion in a 32-week pregnancy. BMJ case reports. 2014;2014:bcr2013202974.

6. Kim SK, Chung JE, Bai SW, Kim JY, Kwon HK, Park $\mathrm{KH}$, et al. Torsion of the pregnant uterus. Yonsei Med J. 2001;42(2):267-9.

7. Nielsen TF. Torsion of the pregnant human uterus without symptoms. Am J Obstet Gynecol. 1981;141(7):838-9.

8. Visser AA, Giesteira MV, Heyns A, Marais C. Torsion of the gravid uterus. Case reports. $\mathrm{Br} \mathrm{J}$ Obstet Gynaecol. 1993;90(1):87-9.

9. Steigrad SJ. Torsion of the gravid uterus. Aust NZ J Obstet Gynaecol. 1987;27(1):66-8.
10. Ulu I, Güneş MS, Kiran G, Gülşen MS. A rare cause of placental abruption: uterine torsion. JCDR. 2016;10(1):QD06.

11. Nicholson WK, Coulson CC, Mc Roy MC, Semelka $\mathrm{RC}$. Case report-pelvic magnetic resonance imaging in the evaluation of uterine torsion. Obstet Gynecol. 1995;5pt2:888-90.

Cite this article as: Tanouti $\mathrm{S}$, Chakri $\mathrm{M}$, Taheri $\mathrm{H}$, Saadi H, Mimouni A. Uterine torsion of 90 degrees: case report. Int J Reprod Contracept Obstet Gynecol 2020;9:1743-5. 